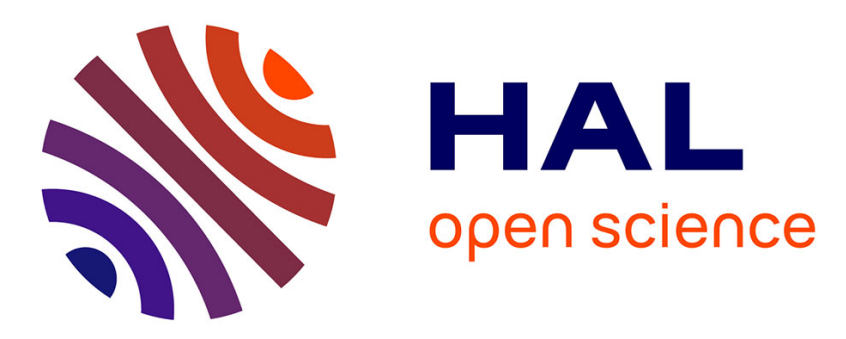

\title{
Numérisation et extension du patrimoine littéraire. Réflexions à propos de " Mauriac en ligne "
}

Jessica de Bideran

\section{To cite this version:}

Jessica de Bideran. Numérisation et extension du patrimoine littéraire. Réflexions à propos de " Mauriac en ligne ". Fabienne Henryot. La fabrique du patrimoine écrit Objets, acteurs, usages sociaux, Presses de l'enssib, pp.115-126, 2020, 10.4000/books.pressesenssib.10587 . hal-02470121

\section{HAL Id: hal-02470121 \\ https://hal.science/hal-02470121}

Submitted on 7 Feb 2020

HAL is a multi-disciplinary open access archive for the deposit and dissemination of scientific research documents, whether they are published or not. The documents may come from teaching and research institutions in France or abroad, or from public or private research centers.
L'archive ouverte pluridisciplinaire HAL, est destinée au dépôt et à la diffusion de documents scientifiques de niveau recherche, publiés ou non, émanant des établissements d'enseignement et de recherche français ou étrangers, des laboratoires publics ou privés. 


\section{Numérisation et extension du patrimoine littéraire.}

\section{Réflexions à propos de "Mauriac en ligne »}

Jessica de Bideran, Université Bordeaux Montaigne - MICA.

Plan :

1. De l'ambiguïté du patrimoine littéraire

2. François Mauriac, en bon gestionnaire sur ces terres...

3. Mauriac en ligne et les nouveaux lieux d'existence du patrimoine littéraire

\section{Mots-clés :}

Patrimoine littéraire, maisons d'écrivain, document, numérisation, collection numérique

Si les sources et ressources en accès libre sur le web sont désormais largement intégrées aux pratiques des chercheurs en Sciences Humaines et Sociales (SHS), nombreux sont les programmes qui s'attellent également à l'élaboration progressive de nouvelles connaissances en s'appuyant au préalable sur la numérisation et la structuration de documents anciens. Moins spectaculaires dans leurs développements informatiques que $d^{\prime}$ 'autres techniques comme la fouille et la visualisation de données, ces pratiques relèvent tout autant des humanités numériques, en ce sens qu'elles participent à une recomposition du paysage académique en s'appuyant sur l'interdisciplinarité et en reconfigurant les relations entre science et société (Dacos et Mounier, 2014).

La numérisation et la diffusion en ligne de ces ressources permettent en effet de faire connaître une partie importante du patrimoine documentaire, jusqu'alors réservée aux seuls usagers des salles de lecture d'archives ou des fonds patrimoniaux des bibliothèques. Une des premières retombées du développement des humanités numériques au sein de la communauté académique se manifeste donc concrètement à travers cette mise en circulation d'objets relevant du patrimoine culturel commun. Mais, au-delà de cette affirmation, dans quelle mesure ce mouvement de numérisation participe-t-il d'une recomposition du processus de patrimonialisation ? Comment les collections patrimoniales sont-elles bousculées par cette numérisation massive des fonds d'archives et de bibliothèques ? Et quel rôle jouent les politiques publiques, culturelles et scientifiques, dans cette recomposition de la patrimonialité, c'est-à-dire de la valeur symbolique accordée à ces objets et aux concepts qu'ils participent à définir (Watremez, 2010) ? 
L'exemple précis des collections mauriaciennes et, plus largement, du travail actuellement mené par l'équipe du projet "Mauriac en ligne " nous permet d'apporter quelques éléments de réponse à ces questions. Les projets qui s'articulent aujourd'hui autour des ressources mauriaciennes, portés par l'Université Bordeaux Montaigne en lien étroit avec le Centre François Mauriac de Malagar (CFMM) et la région Nouvelle-Aquitaine, ainsi que les usages numériques associés ou attendus permettent en effet de repenser ce processus de patrimonialisation des écrits d'écrivains. À partir d'une approche empirique des différentes actions que nous participons à mettre en place, et dans une perspective infocommunicationnelle, nous proposons donc d'interroger le concept de patrimoine littéraire et sa fabrique. Dans cet optique, le numérique est pensé comme une nouvelle " machine à produire des patrimoines "(Gravari-Barbas, 2010), une machine qui le recompose et l'actualise par de nouveaux enjeux de signalement et de connaissance...

\section{De l’ambiguïté du patrimoine littéraire}

Contrairement à ce que pourrait laisser penser les nombreuses et précieuses réserves patrimoniales des bibliothèques françaises, le "patrimoine littéraire " constitue un objet complexe à définir et aux limites difficilement cernables. Si le Code du patrimoine propose aux archives et aux bibliothèques un cadre légal aux fonctions de conservation et de diffusion des documents dont elles ont la charge, nul texte n'officialise en effet une définition du "patrimoine littéraire", à l'image par exemple de celle donnée au « patrimoine archéologique $»^{1}$.

Comment dès lors aborder la fabrique du patrimoine littéraire ? Suivant l'approche développée par Jean Davallon, nous ne traiterons pas ici du patrimoine littéraire en tant que tel mais plutôt de sa patrimonialisation, c'est-à-dire du processus social par lequel les objets, appartenant à un groupe social, une communauté, un collectif ou une nation, changent de statut pour acquérir celui de patrimoine (2002). Contrairement donc à la définition issue de la tradition historienne et qui fait de la perte d'usage et d'une possible disparition physique deux éléments fondamentaux de la patrimonialisation, l'optique info-communicationnelle fait de la réorientation du regard porté sur le bien culturel le principe essentiel de ce processus de reconnaissance.

La fabrique du patrimoine littéraire peut ainsi à la fois être écrite par une histoire des collections patrimoniales des bibliothèques, mais aussi par un examen attentif de toutes les actions mises en place par différents acteurs afin de donner « de la célébrité et de la visibilité » à des écrivains (Jipa, 2015). Politiques, éditeurs, chercheurs et pédagogues participent en effet, selon les époques et de différentes façons, à cette fabrique d'un patrimoine littéraire en sélectionnant des figures d'auteurs dont les œuvres sont

\footnotetext{
${ }^{1}$ Voir le Code du patrimoine, en ligne : https://goo.gl/XPNq5a
} 
considérées comme admirables ou comme représentatives d'un courant artistique et d'une histoire.

Faire reconnaître cette nouvelle valeur assignée à la production littéraire, la faire exister au sein de l'espace social et assurer sa transmission de générations en générations passent dès lors par diverses actions telles que les commémorations nationales, des rééditions plus ou moins luxueuses ou l'intégration de ces textes au sein des programmes scolaires. Et si au cours des $\mathrm{XIX}$ et $\mathrm{XX}$ e siècles, le développement des modèles éditoriaux, de la collection « Les Grands Écrivains Français " aux Livres de Poche (Labbé et Martens, 2015), concourent à la reconnaissance de l'écrivain comme symbole particulier et unificateur de la nation, la récente mais omniprésente référence au patrimoine littéraire dans le socle commun des programmes scolaires atteste d'un désir politique de faire culture commune (Louichon, 2015).

Mais qu'il soit considéré comme un objet scolaire ou comme un objet de délectation, le patrimoine littéraire est ici avant tout pensé comme un texte détaché de "l'objet écrit " (Cavallo et Chartier, 2001) qui lui donne vie, qui permet son appropriation et dont la réception évoluera en fonction des choix éditoriaux, des contextes de lecture et des époques. Apparaît ici toute l'ambiguïté du concept de patrimoine littéraire, où l'œuvre de l'écrivain est érigée en lieux de mémoire intemporel et intangible mais dont les traces, au sens archéologique, sont pour la plupart invisibles puisque conservées au sein des fonds patrimoniaux des bibliothèques et des archives. Le patrimoine littéraire se rapproche en cela du patrimoine immatériel, reconnu comme étant un ensemble de traditions et d'expressions héritées de nos ancêtres et transmises à nos descendants, représentatif d'une culture et d'une communauté, vivant, à l'image de la littérature actualisée à chaque nouvelle lecture, mais nécessitant pour être reconnu des objets matérialisant son existence.

Car si la littérature est bien, par essence, "libérée de l'espace et du temps " (Fabre, 2001), le patrimoine littéraire se matérialise avant tout dans des documents, qu'il s'agisse de manuscrits, de tapuscrits, de correspondances, d'imprimés, de photographies, etc., qui renseignent les différents aspects de l'acte d'écriture mais aussi de la vie intime ou publique de l'écrivain. Le patrimoine littéraire n'existe donc pas a priori en tant que tel, mais est construit comme entité reconnaissable et transférable par tout un ensemble de métiers qui s'articulent autour de la recherche, de la gestion et, enfin, de la valorisation documentaires. Et ce sont bien ces métiers qui assurent toutes les étapes de la chaîne de fabrication du patrimoine littéraire, de la conservation et la transmission dans le temps de ces traces documentaires à la production, à partir de ces traces, de savoirs transmissibles et de significations appropriables par la communauté, assurant ainsi la reconnaissance du statut patrimonial de l'œuvre littéraire. Ainsi le patrimoine littéraire navigue nécessairement entre le régime allographique de la littérature, qui transcende à travers les époques la matérialité du l'objet écrit et de ses réceptions (Genette, 1994), et le régime autographique des objets patrimoniaux qui portent dans leur propre matérialité leur référence au passé qu'ils nous permettent de comprendre (Davallon, 2015).

Installant encore davantage cette nécessaire matérialisation du patrimoine littéraire dans l'espace public et renforçant ainsi ces collections documentaires, quasi muséales, 
conservées par des bibliothèques, s'ajoutent depuis le milieu des années 1990 des lieux monumentaux, réunis aujourd'hui en réseau et reconnus sous l'appellation de " maisons d'écrivains ". Véritable repère spatial de cette mémoire progressivement construite autour de l'auteur (Bonniot, 2016), la maison d'écrivain, tout comme les fonds littéraires, n'occupe plus sa fonction première, utilitaire, d'habitation ou de lieu d'écriture, mais contribue bien à la fabrique de ce patrimoine littéraire en permettant à des publics de rencontrer par des actions culturelles et de manière globale, l'écrivain, ses textes et ses objets légendaires (Gudin de Vallerin, 2010), à l'image du service à eau en opaline verte pieusement conservé à Malagar, en Gironde, et rendu célèbre par son double littéraire tintant à chaque passage de train dans Génitrix. C'est bien sûr par sa présence et sa valorisation, que cet objet, anodin ailleurs, peut donner à la visite de Malagar un caractère de commémoration de l'œuvre romanesque qui n'est plus réduite à une représentation mentale mais prend forme dans une réalité physique. Celui-ci atteste in fine de ce que qui fait ici patrimoine, les romans de François Mauriac reconnus comme patrimoine littéraire...

Mais au-delà de l'anecdote muséographique racontée à chaque visiteur découvrant la maison de François Mauriac, sur quelles traces et quelles valeurs s'est progressivement construit le patrimoine littéraire mauriacien ? Quels objets matériels venus du passé, quelles institutions culturelles et quels savoirs scientifiques ont permis tout à la fois d'ériger ces réalités hétérogènes en un dispositif signifiant permettant de reconnaître le statut patrimonial de cette œuvre littéraire ?

\section{François Mauriac, en bon gestionnaire sur ces terres...}

La signature de François Mauriac s'associe désormais à celles de Montaigne et de Montesquieu sur la façade vitrée de la Bibliothèque municipale de Bordeaux et deux maisons lui ayant appartenue sont, depuis plusieurs années, conservées et gérées par la Région Nouvelle-Aquitaine. Ainsi, le lien entre François Mauriac, sa figure d'écrivain et d'intellectuel français, son œuvre littéraire récompensée en 1952 par le prix Nobel, et le territoire aquitain ne semble plus à démontrer. Cette association résulte cependant de ce long processus de patrimonialisation de la figure de l'écrivain et dont l'ancrage territorial n'est qu'une des valeurs associées à son œuvre.

Dès 1968, en effet, François Mauriac, " en bon gestionnaire de ses écrits " (Baudorre, 2011), lègue à la bibliothèque parisienne Jacques Doucet $^{2}$ un premier ensemble de documents constitué de manuscrits autographes, manuscrits dactylographiés, épreuves corrigées, correspondance et papiers personnels. Ce premier don, voulu et organisé par l'auteur lui-même, atteste du désir de celui-ci de transmettre à la postérité les traces de son activité créatrice, mais aussi de ses divers engagements politiques déjà valorisés par les

\footnotetext{
${ }^{2}$ Bibliothèque publiques patrimoniale consacrée à la littérature française de la seconde moitié du XIX ${ }^{\mathrm{e}}$ siècle à nos jours. Créée par le couturier Jacques Doucet, léguée à l'Université de Paris en 1929, elle relève depuis 1972 de la Chancellerie des Universités de Paris
} 
reprises en volumes de son Journal ainsi que de ses Mémoires. Léguer à cette institution parisienne, reconnue depuis le début $d u X X$ siècle pour la richesse de ses collections patrimoniales dédiées à la littérature, $c^{\prime}$ est en effet provoquer et officialiser une certaine forme de consécration littéraire nationale... Ce don, poursuivi après son décès par sa famille entre 1970 et $1990^{3}$, entérine de fait une première patrimonialisation de l'œuvre mauriacienne, qui change dès lors de statut : bibliothécaires et chercheurs sont désormais les dépositaires de ces documents qu'ils n'ont certes pas produits mais qu'ils travaillent et analysent pour leur richesse historique ou artistique et pour l'intérêt social et culturel que ces derniers représentent pour un nombre plus large de lecteurs, passionnés et publics divers.

Ce premier don signe conséquemment toute l'ambiguïté, déjà évoquée ci-dessus, du patrimoine littéraire qui relève tout à la fois de l'immatériel et du matériel. À l'inverse de la nécessaire rupture mémorielle dans la transmission qui permet de distinguer le processus de patrimonialisation des objets matériels de la simple diffusion d'une mémoire collective (Davallon, 2006), ce geste de l'écrivain n'est en effet pas sans rappeler les procédures aujourd'hui mises en place dans la sauvegarde du patrimoine immatériel... La transmission dans le temps d'objets, ici les traces matérielles de l'œuvre littéraire et de la vie publique de l'écrivain, précède en partie la production de savoirs scientifiques et la reconnaissance patrimoniale de ces objets est assurée en quelques sortes par leur propre créateur... Ce n'est d'ailleurs qu'en 1977 que seront produits les premiers travaux réguliers du Centre d'études et de recherches sur François Mauriac de I'Université Bordeaux Montaigne qui profitera ensuite du centenaire de la naissance de l'écrivain, en 1985, pour publier un premier grand colloque commémoratif.

Car à partir des années 1990, une nouvelle orientation dans le processus de patrimonialisation de l'écrivain François Mauriac semble se profiler, nouvelle orientation qui se dessine parallèlement à la reconnaissance de ce « concept hybride " (Melot, 1997) qu'est la maison d'écrivain. Au centre de plusieurs rapports, journées d'études et colloques, la question des maisons d'écrivains et de leurs rapports avec les fonds littéraires des bibliothèques est alors au cœur des réflexions du Ministère de la Culture, réflexions qui aboutissent en 1996 à une première mise en réseau de ce type bien particulier de monuments. Et si le domaine de Malagar a, pour sa part, fait l'objet d'une donation par les quatre enfants de l'écrivain au Conseil régional d'Aquitaine dès 1985, la création de l'association en charge de sa gestion et les travaux de restauration de la demeure et de son paysage environnant, classés "monument historique » en 1996 du fait même de leur lien avec l'auteur ${ }^{4}$, concourent à l'organisation d'une première saison culturelle en 1999.

\footnotetext{
${ }^{3}$ Par la suite, après le décès de l'écrivain, les acquisitions se poursuivent, d'abord à l'initiative de son fils Claude en 1970, puis de sa femme, Jeanne, entre 1971 et 1972 et divers dons sont enfin réalisés par ses enfants, entre 1990 et 1991, et complètent ce premier corpus parisien qui regroupe des documents datés de 1898 à 1972 et qui s'étalent aujourd'hui sur plus de 12 mètres linéaires.

${ }^{4}$ En raison, comme le signale le texte officiel de classement, " des souvenirs littéraires qui se rattachent à ces lieux ». Pour l'extension aux Jardins de 2013, voir la parution au Journal Officiel en ligne : JORF n0107 du 8 mai 2014 page 7804 , texte $n^{\circ} 31$ : https://www.legifrance.gouv.fr/eli/liste/2014/5/8/MCCC1408858K/jo
} 
Depuis, le public peut venir découvrir le domaine et sa demeure bourgeoise tels que François Mauriac les a connus et les a laissés. Et tandis que les scolaires profitent des diverses actions pédagogiques mises en place par l'équipe chargée de l'animation, des publics variés viennent, à travers ce lieu, ressentir " cette émotion mal définissable que suscite le monument " (Fabre, 2001) et qui donne l'impression de toucher du doigt la sacralité de l'écrivain (Benichou, 1973). Le rappel permanent du rapport étroit unissant cette nature et cette demeure à l'œuvre littéraire, essentiellement romanesque, de François Mauriac est ainsi au centre du récit raconté par l'établissement en charge de ce " haut lieu mauriacien » (Cocula et Llado, 2002).

Son " invention ", au sens archéologique, voulue conjointement par la famille et par les politiques culturelles régionales assure donc la destinée posthume de cet endroit et la construction du mythe de l'écrivain en ses terres... Et pour asseoir cette évidence monumentale et territoriale, le CFMM s'est lancé à partir des années 2000 dans une thésaurisation documentaire ${ }^{5}$ permettant, selon les mots de son directeur d'alors, JeanClaude Ragot, de dynamiser ce lieu en évitant toute nostalgie trop pieuse (Rossignol, 2014) ${ }^{6}$. Aux paysages et mobiliers "malagariens", lieux de vie des romans de l'écrivain, s'ajoutent ainsi de nombreuses pièces d'archives, telles ces photographies familiales ou le prix Nobel reçu en 1952, ou des éditions originales exposées dans le "chai du rouge ", sorte d'espace d'interprétation dédié à l'animation culturelle...

Centre d'étude avec sa bibliothèque de référence, lieu de pédagogie accueillant plusieurs milliers de scolaires, espace de manifestations culturelles mettant en scène une littérature non plus individuelle mais socialisée, réserve d'archives renseignant l'intimité de

\footnotetext{
${ }^{5}$ À ce jour cette collection regroupe près de 28000 pièces documentaires permettant d'atteindre non seulement l'écrivain mais aussi l'homme dans son intimité. Ce fonds s'est progressivement constitué grâce à de nombreux dons effectués par la famille, des experts et spécialistes de François Mauriac - le dernier don en date a ainsi été réalisé par l'Université Bordeaux Montaigne en septembre 2016 et concernait des archives de presse - ainsi que par des achats réguliers effectués par la direction. Après un inventaire et un récolement minutieux de ce fonds, le centre de documentation a catégorisé les ressources selon 3 types différents : 1) les archives écrites privées et thématiques regroupent près de 15000 documents (ou groupes de documents) écrits (correspondance, agendas, notes diverses, etc.) ; 2) les archives concernant la presse regroupent le courrier des lecteurs (1900 lettres) et des coupures de presse publiées sur François Mauriac et/ou sa famille (plus de 2600 articles) ; 3) les archives photographiques regroupent environ 5000 clichés (albums familiaux de Malagar, clichés de Jeanne-François Mauriac, photographies professionnelles, etc.) auxquels s'ajoute un don récent de plus de 3.500 clichés familiaux.

${ }^{6}$ À noter que ces acquisitions se font en concertation avec la Bibliothèque municipale de Bordeaux qui conserve et acquière également des ressources mauriaciennes depuis 1973 suite à un don de Jeanne Mauriac, épouse de l'écrivain. Celle-ci offre alors les manuscrits du discours que son mari a prononcé pour ses 80 ans, au Grand-Théâtre de Bordeaux en 1965. Dans les années qui suivent, la famille Mauriac fait don à la bibliothèque d'importants manuscrits littéraires et d'archives familiales et aujourd'hui, ce fonds est l'ensemble littéraire contemporain le plus important de la bibliothèque. Le fonds, tel qu'il est traité actuellement, totalise environ 2000 pièces, soit plus de 1500 lettres, presque 400 manuscrits d'œuvres (romans, articles, discours, etc.), plusieurs dizaines d'éditions originales et rares, plus de 200 pièces de papiers personnels (cahiers d'écolier, cartes et faire-part, etc.), environ 20 photographies (prises de vues officielles, cartes postales et photographies plus intimes), 10 publications originales d'articles dans des périodiques d'époque, environ 40 coupures de presse et plusieurs estampes. II reste à traiter : environ 150 coupures de presse, 50 photographies, plusieurs centaines de lettres, une vingtaine de manuscrits de Raymond Mauriac, quelques dossiers d'exposition de la bibliothèque.
} 
l'écrivain ${ }^{7}$; Malagar a progressivement vu sa valeur patrimoniale se déplacer, de sa seule valeur d'existence à une valeur d'usages renouvelant les possibilités de réception de la littérature (Thuillas, 2009). Désormais ancré sur un territoire et dans une offre d'activités culturelles bien identifiées, le patrimoine littéraire mauriacien se dote ainsi de nouveaux publics, qui dépassent les seuls amateurs ou passionnés et qui appréhendent toute la richesse de l'écrivain, auteur internationalement reconnu et intimement attaché à ses terres...

Entre site et fonds documentaire, le CFMM, à l'image de cet ensemble plus large que représentent les maisons d'écrivain, a progressivement acquis une reconnaissance publique qui permet de redonner vie à François Mauriac en faisant de son œuvre et de son activité une partie d'un tout plus large, ce fameux patrimoine littéraire. Mais parallèlement à cette assise territoriale et monumentale, la propagation désormais massive des outils et réseaux numériques entraîne la création de nouveaux espaces d'existence et de circulation des patrimoines qui ne sont pas sans conséquences sur ce concept même...

\section{Mauriac en ligne et les nouveaux lieux d'existence du patrimoine littéraire}

Alors que sur certains territoires des initiatives numériques ont été conduites pour " géo-référencer » des extraits d'œuvres littéraires ainsi accessibles en mobilité (Thuillas, 2010), d'autres projets s'inscrivent dans le mouvement massif de mise en ligne d'archives et, plus largement, d'artefacts documentaires. Depuis 2004, la région Aquitaine soutient ainsi la mise en œuvre d'un vaste chantier de numérisation de ressources patrimoniales conservées par les archives, bibliothèques, musées et centres de documentation de nombreuses collectivités et institutions (Sibers, 2011). C'est au sein de ce chantier documentaire et technique, en perpétuelle évolution, que s’est inséré le projet « Mauriac en ligne ».

Sur le terrain, celui-ci a ainsi débuté dès 2011 avec l'inventaire et le récolement, à l'aide d'outils bibliographiques ${ }^{8}$, des quelque 3000 textes de presse rédigés par François Mauriac entre 1905 et 1970 . Cette étape de localisation franchie, et après une première expérimentation d'édition numérique réalisée avec l'Université d'Exeter au format XML-TEI ${ }^{9}$,

\footnotetext{
${ }^{7}$ À cette première liste, on pourrait rajouter plus récemment la fonction de résidence d'artistes pour le chalet Mauriac situé sur la commune girondine de Saint-Symphorien, seconde demeure de l'écrivain aujourd'hui propriété de la région Nouvelle-Aquitain ; voir en ligne : http://chaletmauriac.aquitaine.fr/

${ }^{8}$ Voir à ce propos Goesch, Keith. Écrits de François Mauriac parus dans la presse. Vol. 1 et 2. Paris : Lettres modernes Minard, 2005. Granger, Laurence. L'Esprit critique dans l'œuvre journalistique de François Mauriac 1905-1970. Thèse de $3^{\mathrm{e}}$ cycle. Université Bordeaux Montaigne, 2006.

${ }^{9}$ Celle-ci est toujours accessible en ligne http://mauriac.ex.ac.uk/. La confrontation avec le système éditorial retenue par l'équipe bordelaise, accessible depuis http://mauriac-en-ligne.u-bordeaux-montaigne.fr/, permet de souligner les différences de traitement documentaire effectués. Si l'on peut en effet souligner la richesse de l'annotation et de l'appareil critique de la première édition on ne peut que s'interroger sur l'absence de réflexion sur les usages de ces textes...
} 
divers fonds ont été sollicités à partir de 2014 pour compléter la collection réunie par le Service Commun de Documentation de l'Université Bordeaux Montaigne et obtenir ainsi au format numérique tous les articles parus, à quelques exceptions près, entre 1905 et 1947. Pour des questions juridiques, seule cette première production journalistique peut en effet pour l'instant être diffusée en ligne en libre accès puisqu'appartenant au domaine public. Repérage, collecte, récolement et numérisation effectués, les articles ont ensuite été structurés au sein d'une base de données publique construite selon quatre grands principes éditoriaux: 1) l'exhaustivité en recherchant l'intégralité des articles publiés par François Mauriac ; 2) le respect de la spécificité de l'ensemble des textes, hétérogène et fragmenté, issu d'une production périodique qui a souvent fait l'objet de reprises des mains mêmes de l'écrivain par la suite ; 3) un accès libre, ouvert et gratuit ; 4) une diffusion enrichie à partir d'une réflexion sur les usages et les lecteurs visés.

Au printemps 2018, la base de données construite grâce au CMS Omeka réunit ainsi plus de 900 textes transcrits et décrits au format international Dublin Core (Bideran, 2016), soit plus de $97 \%$ de cette première tranche, 25 textes restant malheureusement introuvables. Interrogeables via les métadonnées ou consultables selon les modalités d'enrichissement retenus par l'équipe scientifique, tels que les mots-clés ou l'accès cartographique, ces derniers sont également accessibles depuis deux portails, Aquitaine cultures connectées et Isidore, qui moissonnent les métadonnées grâce au protocole d'échange OAI-PMH. Les circulations et réceptions possibles de ces textes reposent donc en grande partie sur l'indexation mise en place et résultent in fine d'un travail de structuration construit par les chercheurs à partir de leur analyse des textes. L'exploitation de mots-clés libres, plutôt que le recours à un langage contrôlé, construit ainsi un univers sémantique mauriacien incarné, mêlant des noms propres (André Gide, Jacques Rivière, Georges Duhamel, etc.), des noms communs (grâce, amour, enfance, etc.), des locutions (acteurs et actrices, littérature engagée, etc.) ou encore des adjectifs (mufle, immonde, débauché, etc.) qui renouvelle l'approche globale de l'œuvre littéraire en dépassant sa dimension intime, purement romanesque ou aquitaine, pour envisager plus globalement ses divers engagements publics et politiques...

La numérisation ne se réduit donc pas à un simple travail de reproduction des textes... Affranchis de leur milieu d'origine, le périodique, et dépassant les anciennes reprises éditoriales, ces objets numériques sont reconfigurés par un jeu de structuration sémantique qui autorise la mise en place d'une nouvelle «fiction » (Merzeau, 2004) autour du patrimoine littéraire mauriacien. Du point de vue de la fabrique patrimoniale, il s'agit en effet d'attribuer à ces objets numériques et, plus largement, au patrimoine littéraire mauriacien, de nouvelles significations qui doivent ensuite être transmises à la communauté qui consent à cette conservation. La patrimonialisation de la production littéraire et journalistique de l'écrivain François Mauriac entre donc ici dans une nouvelle phase qui se concrétise dans un dispositif numérique singulier (Bonnacorsi, 2015) imbriquant tout à la fois, et c'est une nouveauté, la production scientifique et la circulation médiatique de ces nouvelles significations. Cette mise en patrimoine élabore en effet parallèlement de nouvelles pratiques de conservation, d'étude et de transmission qui sont encore et très 
concrètement en cours d'édification et de reconnaissance par l'ensemble des acteurs travaillant sur ce patrimoine littéraire.

Ce travail d'identification et de normalisation sémantique et la récente multiplication des collections numériques mauriaciennes posent effectivement de nouveaux défis aux membres du projet. Parallèlement au programme universitaire, le CFMM a en effet entrepris diverses actions de numérisation de ses archives. Photographies, coupures de presse et correspondances sont ainsi en cours de conversion numérique afin, dans un premier temps, de pouvoir être consultées sur place au sein du centre de documentation et dans le cadre de projets de recherche et/ou de valorisation. De même, et bien qu'aucune numérisation massive de ce fonds ne soit encore envisagée, une meilleure identification du fonds Mauriac à la Bibliothèque Municipale de Bordeaux est en cours de réalisation par le service patrimonial. Or, toutes ces collections appartiennent au même patrimoine littéraire et seule une véritable mise en réseau devrait permettre de dépasser les logiques institutionnelles « qui sont autant d'obstacles à la recherche et d'injures à l'histoire » (Lignac, 2003).

Car l'une des principales forces de la collection numérique par rapport à la collection physique est sa capacité à dépasser les limites institutionnelles par des procédures d'interrogation à distance et d'interopérabilité qui facilite sa circulation et sa visibilité. Or, ces différents programmes de numérisation et d'identification, autonomes dans leurs objectifs et leurs objets puisque relevant de structures différentes, convergent évidemment par leurs contenus et leurs sujets ainsi que par leurs publics (chercheurs, érudits, scolaires, amateurs...) et donc, assez logiquement, par leurs usages. Des interrogations communes et structurantes pour l'avenir telles que la description des fonds pour une consultation croisée, l'archivage pérenne des données ou l'éditorialisation permettant de faire vivre ces ressources au sein de l'espace public du web doivent dès lors être au cœur du processus de patrimonialisation. Seul un enrichissement documentaire concerté des corpus numériques mauriaciens et la mise en place d'un écosystème commun de consultation et de valorisation permettra ainsi « de recréer virtuellement un ensemble cohérent, construit à partir de contributions documentaires dispersées, mais dont le regroupement fait sens " (Bermès et Martin, 2010)...

\section{Conclusion}

Auparavant seulement lisible à travers des supports éditoriaux, devenu visitable par l'intermédiaire des maisons d'écrivain, le patrimoine littéraire est en passe de devenir encore plus visible grâce aux outils et réseaux numériques. En cela, il adopte les traits de l'ensemble des objets patrimoniaux dont les fac-similés numériques sont aujourd'hui accessibles pour tout le monde partout et tout le temps. Cette circulation s'inscrit, voire confirme, la double dimension de transmission et de projet du patrimoine qui est avant tout un instrument de construction d'une identité et d'un imaginaire communs, donc un instrument de lien social. Et si la transmission de celui-ci reste en partie centrée sur l'usage de l'objet dans l'histoire, les projets de médiation impliquant une reconnaissance identitaire ou territoriale sont de plus en plus mobilisés. La numérisation n'échappe pas à cette 
injonction en produisant de nouvelles collections virtuelles sur lesquelles se cristallisent des enjeux politiques et institutionnels qu'il convient toutefois de dépasser.

Pour exprimer toutes les potentialités de diffusion et de réception de ces collections numériques, de nombreuses voies méritent d'être explorées pour mieux cerner les monuments documentaires que nous participons à édifier (Welger-Barboza, 2012). Et l'extension du patrimonialisable à laquelle participent les humanités numériques doivent sans aucun doute s'accompagner de recherches portant plus spécifiquement sur les réceptions de ces artefacts numériques, de leur "muséographie ", ou mise en exposition, en passant par leurs publics et les usages possibles (scientifiques, pédagogiques ou médiatiques) à travers des outils d'exploration et d'évaluation... C'est en tout cas à quoi s'attelle désormais l'équipe réunie autour de "Mauriac en ligne » dans le cadre d'un programme régional plus large, e-can@, «éditer les corpus d'auteurs en NouvelleAquitaine ".

\section{Bibliographie :}

Baudorre, Philippe. "De Mauriac à Mauriac, la seconde vie des textes de presse", Nouveaux Cahiers François Mauriac, n¹9, 2011 : 83-104.

Benichou, Paul. Le Sacre de l'écrivain, José Corti 1973.

Bermès, Emmanuelle et Martin, Frédéric. "Le concept de collection numérique ". Bulletin des bibliothèques de France (BBF), $\mathrm{n}^{\circ} 3,2010$ : http://bbf.enssib.fr/consulter/bbf2010-03-0013-002

Bideran, Jessica (de). « Du fragment daté au corpus patrimonialisé : numérisation et muséalisation de l'article de presse mauriacien ». Études digitales, $n^{\circ} 1$, $2016: 125-142$.

Bonaccorsi, Julia. "Quelle réflexivité médiatique pour les Humanités Numériques ? La numérisation des manuscrits littéraires ". Les Enjeux de l'information et de la communication, $n^{\circ} 2,2015: 83-97$.

Bonniot, Aurore. Imaginaire des lieux et attractivité des territoires. Une entrée par le tourisme littéraire: Maisons d'écrivain, routes et sentiers littéraires. Thèse, Université Blaise Pascal - Clermont-Ferrand II, 2016 : https://tel.archives-ouvertes.fr/tel-01517269/document

Cavallo, Guglielmo, et Roger Chartier. Histoire de la lecture dans le monde occidental. Paris : Seuil, 2001.

Cocula, Bernard et Llado, Astrid. "Malagar, terre d'éternité ». Bulletin de la Fédération des maisons d'écrivain et des patrimoines littéraires, $\mathrm{n}^{\circ} 7,2002: 8-11$.

Cooke, Paul. "Vers une édition électronique de l'œuvre journalistique de François Mauriac (1937-38) : premiers bilans », Nouveaux Cahiers François Mauriac, n¹9, 2011 : 7382. 
Dacos, Marin et Mounier, Pierre. Humanités numériques : État des lieux et positionnement de la recherche française dans le contexte international, Institut Français, 2014 : http://www.institutfrancais.com/sites/default/files/if humanites-numeriques.pdf

Davallon, Jean. "Mémoire et patrimoine : pour une approche des régimes de patrimonialisation ». Mémoire et nouveaux patrimoines [en ligne]. Marseille : OpenEdition Press, 2015 : http://books.openedition.org/oep/444

Davallon, Jean. Le don du patrimoine, une approche communicationnelle de la patrimonialisation, Paris : Lavoisier - Hermes science, 2006.

Davallon, Jean. "Comment se fabrique le patrimoine ? ". Sciences humaines, horssérie $n^{\circ} 36,2002: 74-77$.

Fabre, Daniel. " Maison d'écrivain ". Le Débat, n¹15, 2001 : 172-77, https://doi.org/10.3917/deba.115.0172

Genette, Gérard. L'œuvre de l'art, Immanence et transcendance, Paris: Poétique/Seuil, 1994.

Gravari-Barbas, Maria. "Le tourisme, un formidable producteur d'espaces urbains ", dans La France, une géographie urbaine. Paris, Armand Colin, 2010 : 325-336.

Gudin de Vallerin, Gilles. "Conserver l'esprit de l'écrivain dans tous ses états ". Bulletin des bibliothèques de France (BBF), 2010 : http://bbf.enssib.fr/consulter/bbf-2010$\underline{03-0040-007}$

Jipa, Dragos. " Le directeur de collection comme " auteur ». Jean-Jules Jusserand et "Les Grands Écrivains Français » (1887-1913) ». Mémoires du livre / Studies in Book Culture 7, n¹, 2015 : https://doi.org/10.7202/1035760ar

Labbé, Mathilde, et David Martens. "Les collections de monographies illustrées de poche. Une fabrique collective du patrimoine littéraire (XIX $-\mathrm{XXI}^{\mathrm{e}}$ siècles) ". Mémoires du livre / Studies in Book Culture 7, n¹, 2015 : https://doi.org/10.7202/1035759ar

Lignac, Florence. "Maisons d'écrivain \& patrimoines littéraires ». Bulletin des bibliothèques de France (BBF), n², 2003 : 48- 51.

Louichon, Brigitte. "Le patrimoine littéraire : un enjeu de formation ". Tréma, n43, 2015 : $\underline{\text { https://doi.org/10.4000/trema.3285. }}$.

Melot, Michel. "Collections littéraires, maisons d'écrivains et bibliothèques ". Bulletin des bibliothèques de France (BBF), 1997 : http://bbf.enssib.fr/consulter/bbf-199702-0028-004.

Merzeau, Louise. «Malraux metteur en page ». In Malraux metteur en page, 2004 : 64-79, <halshs-00838754>

Rossignol, Lorraine. "L'écrivain, ce nouveau musée ». Télérama, publié le 14/07/2014 et mis à jour le 01/02/2018 en ligne : http://www.telerama.fr/livre/l-ecrivain-cenouveau-musee,114728.php 
Sibers, Jean-François. "Banque numérique du savoir en Aquitaine ". Bulletin des bibliothèques de France (BBF), n4, 2011 : 50-56.

Thuillas, Olivier. "GéoCulture : le Limousin vu par les artistes ", L'Observatoire, n³7, 2010 : https://www.cairn.info/revue-l-observatoire-2010-2-page-82.htm

Thuillas, Olivier. Les Nouveaux Modes de valorisation du patrimoine et des sites littéraires : de la valeur d'existence à la valeur d'usage. Mémoire de recherche en Master, Université Pierre Mendès France de Grenoble, 2009.

Watremez, Anne. "Les chercheurs ne patrimonialiseraient-ils pas aussi ? ». Culture \& Musées, n¹6, 2010 : https://doi.org/10.3406/pumus.2010.1567

Welger-Barboza, Corinne. "Le site André Breton - Un monument-document ". L'Observatoire critique (blog), 2012 : https://observatoire-critique.hypotheses.org/1252 